\title{
Characterization of the structure and dynamics of amyloidogenic variants of human lysozyme by NMR spectroscopy
}

\author{
AARON K. CHAMBERLAIN, ${ }^{1,3}$ VERONIQUE RECEVEUR, ${ }^{1,4}$ ANDREW SPENCER, ${ }^{1,2}$ \\ CHRISTINA REDFIELD ${ }^{1}$ AND CHRISTOPHER M. DOBSON ${ }^{1,5}$ \\ ${ }^{1}$ Oxford Centre for Molecular Sciences, University of Oxford, New Chemistry Laboratory, Oxford OX1 3QT, UK \\ ${ }^{2}$ Institute of Food Research, Norwich Research Park, Colney, Norwich NR4 7UA, UK
}

(Received July 11, 2001; Final Revision September 1, 2001; AcCEPTEd September 12, 2001)

\begin{abstract}
The structures and dynamics of the native states of two mutational variants of human lysozyme, I56T and D67H, both associated with non-neuropathic systemic amyloidosis, have been investigated by NMR spectroscopy. The ${ }^{1} \mathrm{H}$ and ${ }^{15} \mathrm{~N}$ main-chain amide chemical shifts of the I56T variant are very similar to those of the wild-type protein, but those of the $\mathrm{D} 67 \mathrm{H}$ variant are greatly altered for 28 residues in the $\beta$-domain. This finding is consistent with the X-ray crystallographic analysis, which shows that the structure of this variant is significantly altered from that of the wild-type protein in this region. The ${ }^{1} \mathrm{H}-{ }^{15} \mathrm{~N}$ heteronuclear NOE values show that, with the exception of V121, every residue in the wild-type and I56T proteins is located in tightly packed structures characteristic of the native states of most proteins. In contrast, D67H has a region of substantially increased mobility as shown by a dramatic decrease in heteronuclear NOE values of residues near the site of mutation. Despite this unusual flexibility, the D67H variant has no greater propensity to form amyloid fibrils in vivo or in vitro than has I56T. This finding indicates that it is the increased ability of the variants to access partially folded conformations, rather than intrinsic changes in their native state properties, that is the origin of their amyloidogenicity.
\end{abstract}

Keywords: NMR; lysozyme; mutant; backbone dynamics; amyloid fibrils

Supplemental material: See www.proteinscience.org for tables containing the assignments and NOE values of the three lysozyme proteins.

Reprint requests to: Christopher M. Dobson, Department of Chemistry, University of Cambridge, Lensfield Road, Cambridge CB2 1EW, UK; e-mail: cmd44@cam.ac.uk; fax: 44-1223-763418.

${ }^{3}$ Present address: Molecular Biology Institute, University of California at Los Angeles, Boyer Hall, 611 E. Charles E. Young Drive, Box 951570, Los Angeles, CA 90095-1570, USA.

${ }^{4}$ Present address: AFMB-CNRS 31 Chemin Joseph Aiguier, 13402 Marseille cedex 20, France.

${ }^{5}$ Present address: Department of Chemistry, University of Cambridge, Lensfield Road, Cambridge CB2 1EW, UK.

Abbreviations: I56T, human lysozyme with isoleucine at position 56 substituted by threonine; $\mathrm{D} 67 \mathrm{H}$, human lysozyme with aspartate at position 67 substituted by histidine; wt, wild type; NMR, nuclear magnetic resonance; NOE, nuclear Overhauser effect; NOESY, NOE spectroscopy; COSY, correlated spectroscopy; HSQC heteronuclear single quantum coherence; 2D, two-dimensional; 3D, three-dimensional; HN, backbone amide hydrogen; $\mathrm{H}_{\alpha}$, alpha hydrogen; $\mathrm{C}_{\alpha}$, alpha carbon.

Article and publication are at http://www.proteinscience.org/cgi/doi/ $10.1101 / \mathrm{ps} .28101$.
Amyloid fibrils are highly ordered protein aggregates that are associated with a variety of pathological conditions known collectively as amyloidoses. Prominent among these conditions are Alzheimer's disease and the transmissible spongiform encephalopathies, or prion, diseases such as Creutzfeld-Jakob disease and scrapie (Pepys 1994; Prusiner 1997; Serpell et al. 1997; Dobson 2001). Despite the sequence and structural diversity of the soluble forms of the proteins that form these aggregates in vivo, when incorporated into amyloid fibrils the proteins share a common cross- $\beta$ structure (Sunde and Blake 1998). In order for the fibrils to form, the protein involved must unfold from its native state to an unstructured or partially structured conformation in an environment where controlled self-association can take place (Chiti et al. 1999; Koo et al. 1999; Lansbury 1999). 

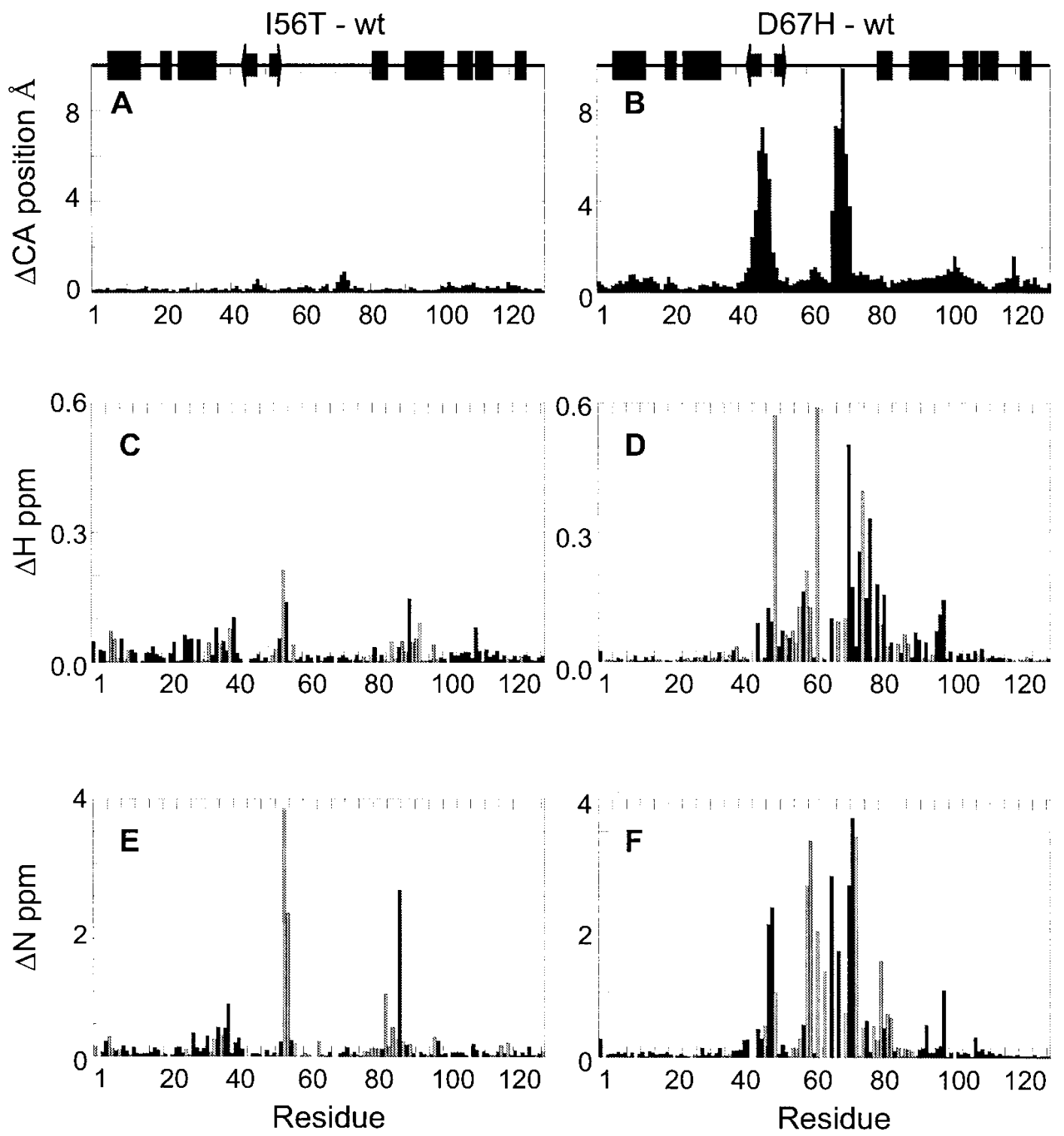

Fig. 1. The effects of the I56T and D67H mutations on the X-ray structure and NMR chemical shifts of human lysozyme. $(A, B)$ The differences in $\mathrm{C}_{\alpha}$ positions from the X-ray structure of the two variants (Booth et al. 1997). (C,D) The differences in the chemical shifts of the backbone ${ }^{1} \mathrm{HN}$ resonances at $37^{\circ} \mathrm{C}$ are shown as black bars (positive values) and gray bars (negative values). $(E, F)$ The differences in the chemical shifts of the backbone ${ }^{15} \mathrm{~N}$ resonances at $37^{\circ} \mathrm{C}$ are shown. At the top of $A$ and $B$, the secondary structural elements are shown: helices (bars) and $\beta$-sheets (arrows). ( $A, C, E)$ I56T minus wild type. $(B, D, F)$ D67H minus wild type.

The human protein lysozyme has been found to form amyloid fibrils in vivo in individuals from families carrying one of two nonconservative point mutations in the lysozyme gene (Pepys et al. 1993). The affected individuals of these families have non-neuropathic systemic amyloidosis in which large quantities, often kilograms, of aggregated protein accumulate in organs such as the liver, kidney, and spleen. The fibrils extracted from the livers of the affected individuals have a substitution of isoleucine 56 to threonine (I56T) or aspartate 67 to histidine $(\mathrm{D} 67 \mathrm{H})$ relative to the wild-type protein. The native structure of human lysozyme has two distinct structural domains denoted $\alpha$ and $\beta$. The
I56T mutation site lies in the interface between the $\alpha$ - and $\beta$-domains, whereas the D67H mutation is located in a long loop within the $\beta$-domain.

The properties of the amyloidogenic variants I56T and D67H have been studied and compared to those of the wildtype protein in order to examine how these mutations affect the structure, folding, and stability of the protein (Funahashi et al. 1996; Booth et al. 1997; Canet et al. 1999; MorozovaRoche et al. 2000). The variant proteins recovered and refolded from ex vivo fibrils have similar activities to the wild-type enzyme ( $k_{\mathrm{cat}}$ and $K_{\mathrm{M}}$ values are within a factor of 2 ), and mass spectrometric data show that the fibrils contain 
full-length protein and that all four disulfide bonds remain intact.

Crystallographic studies show that the native structure of I56T contains no deviations in the $\mathrm{C}_{\alpha}$ position from that of the wild-type protein that are greater than $1 \AA$ (Fig. 1A; Booth et al. 1997). The structure of native D67H, however, shows extensive differences from that of the wild-type protein in the $\beta$-domain. The D67H substitution disrupts a hydrogen-bond network consisting of Y54, D67, K69, and T70 in the long loop located within the $\beta$-domain and the short loop between the two short $\beta$-strands that form a small, antiparallel $\beta$-sheet. These two loops undergo a concerted movement away from each other, resulting in deviations in $\mathrm{C}_{\alpha}$ positions from the structure of the wild-type protein of up to $10 \AA$ in the vicinity of residues G48 and T70 (Fig. 1B). These two mutations, therefore, result in substantially different perturbations to the native structures. Nevertheless, the two variants both have a similar decrease in the stability of the native state and a similar increase in the propensity to form amyloid fibrils (Booth et al. 1997). At $\mathrm{pH} 2.0$, amyloid fibrils can readily be prepared from the mutants at $37^{\circ} \mathrm{C}$, compared to $57^{\circ} \mathrm{C}$ used with the wild-type protein (Morozova-Roche et al. 2000). These findings prompted us to investigate the structural and dynamic properties of the native proteins in solution by means of NMR spectroscopy.

\section{Results and Discussion}

\section{Chemical shift changes for I56T and D67H human lysozymes}

The chemical shifts of the two mutational variants were compared to those of the wild-type protein in order to probe the extent to which the mutations affect the structure of their native states. Under the solution conditions used in this work $\left(\sim 1 \mathrm{mM}\right.$ protein in $90 \% \mathrm{H}_{2} \mathrm{O} / 10 \% \mathrm{D}_{2} \mathrm{O}$ at $\left.\mathrm{pH} 5.0\right)$, the resonance line widths of the two variants are similar to those of the wild-type protein, indicating that they are monomeric. Small amounts of precipitation were, however, visible when the samples were left standing for periods in excess of $4 \mathrm{wk}$. Spectra of the wild-type protein collected under our solution conditions ( $\mathrm{pH} 5.0$ and $20^{\circ} \mathrm{C}$ or $37^{\circ} \mathrm{C}$ ) were assigned by comparison to previously published spectra of human lysozyme (Boyd et al. 1985; Redfield and Dobson 1990; Ohkubo et al. 1991) and confirmed by $\mathrm{HN}-\mathrm{HN}$ and $\mathrm{HN}-\mathrm{H}_{\alpha}$ connectivities determined from ${ }^{15} \mathrm{~N}$ NOESY-HSQC experiments.

The changes in the ${ }^{1} \mathrm{H}$ and ${ }^{15} \mathrm{~N}$ chemical shifts of the main-chain amide resonances of the variant lysozymes relative to those of the wild-type protein are shown in Figure 1. In the case of I56T (Fig. 1C,E), the spectral assignments could readily be made by direct comparison of HSQC and COSY spectra with similar spectra of wild-type human ly- sozyme; only four amide proton chemical shifts differ by $>0.1 \mathrm{ppm}$ from the wild-type values. These resonances are those of residues A42, T56, F57, and A92, all of which are spatially close to the site of the mutation, having at least one atom within $5.5 \AA$ of its backbone $\mathrm{N}$ atom.

The changes in chemical shift caused by the $\mathrm{D} 67 \mathrm{H}$ mutation are, however, considerably more extensive. The spectral assignments of the residues in the $\alpha$-domain of the variant were achieved by direct comparison with the assignments of the wild-type protein. Most of the assignments of residues in the $\beta$-domain, however, were achieved by analysis of $3 \mathrm{D}{ }^{15} \mathrm{~N}$ NOESY-HSQC experiments. Of particular importance were observations of sequential $\mathrm{HN}-\mathrm{HN}$ and $\mathrm{HN}-\mathrm{H}_{\alpha}$ NOE connectivities from F3-E4 to C65-N66 and G72-A73 to V130. Residue 71 is a proline and breaks the connectivity required for sequential assignments; indeed, resonances of G68 and T70 could only be assigned tentatively on the basis of HSQC and COSY spectra. An additional complexity is that the G68 cross peak is of very low intensity in the HSQC spectrum as a result of line broadening. The backbone ${ }^{1} \mathrm{HN}$ and ${ }^{15} \mathrm{~N}$ resonances at the site of the mutation (H67) and of one other residue (K69) could not be detected, probably as a result of extreme line broadening. The increased line widths of all these resonances are likely to arise from conformational fluctuations on the millisecond to microsecond time scale.

The ${ }^{1} \mathrm{H}$ and ${ }^{15} \mathrm{~N}$ chemical shift values of the main-chain amide groups are substantially perturbed from those of the wild-type protein for residues $48-53$ and $60-82$ of $\mathrm{D} 67 \mathrm{H}$ (Fig. 1D,F). These residues are all located in the $\beta$-domain, in a region where the $\mathrm{X}$-ray diffraction data indicate significant movement of their atomic position as a consequence of the mutation (Booth et al. 1997). The crystal structure of $\mathrm{D} 67 \mathrm{H}$ reveals that the mutation disrupts a series of hydrogen bonds in the $\beta$-domain, resulting in a movement of the $\beta$-sheet residues and the long loop containing residues $66-74$, as described above. The $\mathrm{C}_{\alpha}$ atoms of 12 residues (Y45-R50 and G68-A73) are perturbed substantially (by $>2.0 \AA$ ) from their positions in the wild-type structure. Approximately 30 residues located in this region have been found to have significant perturbations to their chemical shifts (of $>0.1 \mathrm{ppm}$ in ${ }^{1} \mathrm{H}$ or $0.5 \mathrm{ppm}$ in ${ }^{15} \mathrm{~N}$ ); these residues are G48-D52 and T60-S82. Although the detailed nature of these changes cannot be defined from the present NMR analysis, this observation indicates that there are subtle changes in the environment of residues extending over more of the $\beta$-domain than might be anticipated from the $\mathrm{X}$-ray data.

\section{Internal dynamics of wild-type, I56T, and D67H human lysozymes}

To study the effects of the amyloidogenic mutations on the fast-time-scale dynamics of human lysozyme, we have mea- 
sured the backbone ${ }^{1} \mathrm{H}-{ }^{15} \mathrm{~N}$ heteronuclear nuclear Overhauser effects (NOEs) for both the variants and the wildtype protein at $20^{\circ} \mathrm{C}$ and $37^{\circ} \mathrm{C}$. Of the 130 residues in each protein, NOE values could be measured for 90 and 106 residues at $20^{\circ} \mathrm{C}$ and $37^{\circ} \mathrm{C}$, respectively, for the wild-type protein. At the same temperatures, 115 and 113 NOE values could be measured for I56T, and 109 and 112 NOE values for $\mathrm{D} 67 \mathrm{H}$, respectively. The different numbers of measured NOE values result from the differing extents of resonance overlap in the various spectra. For all three proteins, the NOE values at $20^{\circ} \mathrm{C}$ and $37^{\circ} \mathrm{C}$ are generally similar, indicating that their internal motions are largely unaffected by an increase from room to physiological temperature (Fig. 2).

As shown in Figure 2A, the wild-type protein has consistently high values for the heteronuclear NOE of its constituent residues, as expected for a native protein of this size having a close-packed cooperative structure (Wuthrich 1986; Eliezer et al. 1998). Residues N44, Q86, and Q104 have NOE values significantly lower (between 0.73 and 0.74 at $37^{\circ} \mathrm{C}$ ) than any of the remaining residues with the exception of V121 and the C-terminal residue V130. These three residues are all in surface-exposed loops and also have lower NOE values in the two amyloidogenic variants. V121 is the only residue with an NOE value $<0.7$; it has an NOE value of 0.62 at $20^{\circ} \mathrm{C}$ and 0.64 at $37^{\circ} \mathrm{C}$. The analogous residue in hen egg white lysozyme, V120, does not show this low NOE value, although a single, low NOE value $(0.28)$ is observed in the protein, in this case for S85 (Buck et al. 1995). V121 is at the beginning of the C-terminal helix of lysozyme. Its main-chain torsion angles determined from the crystal structure are in the core $\alpha$-region of the Ramachandran plot as shown by Procheck (Collaborative Computational Project 4, 1994), and its crystallographic B factors are similar to those of the surrounding residues. The residue is, however, $84 \%$ exposed as calculated with the Environments program of James Bowie (University of California at Los Angeles). Similarly, S85 in hen egg white lysozyme is in the core $\alpha$-region of the Ramachandran plot, is located at the beginning of a $3_{10}$ helix, and is $54 \%$ exposed to solvent. Therefore, although neither of these residues has unusual characteristics in the crystal structures of the proteins, they appear to undergo local motions in solution that are not propagated to neighboring residues.

The two amyloidogenic variants of human lysozyme, like the wild-type protein, show low NOE values for N44, Q86, Q104, and particularly V121. Indeed, the heteronuclear NOE values of I56T are all closely similar to those of the wild-type protein throughout the sequence. Even around the site of the mutation, the NOE ratios remain above 0.7 and do not provide evidence of any detectable increase in flexibility relative to the wild-type protein. The situation for D67H is, however, very different from that for I56T, as the NOE data indicate a number of large changes to the internal dynamics of the variant relative to the wild-type protein.

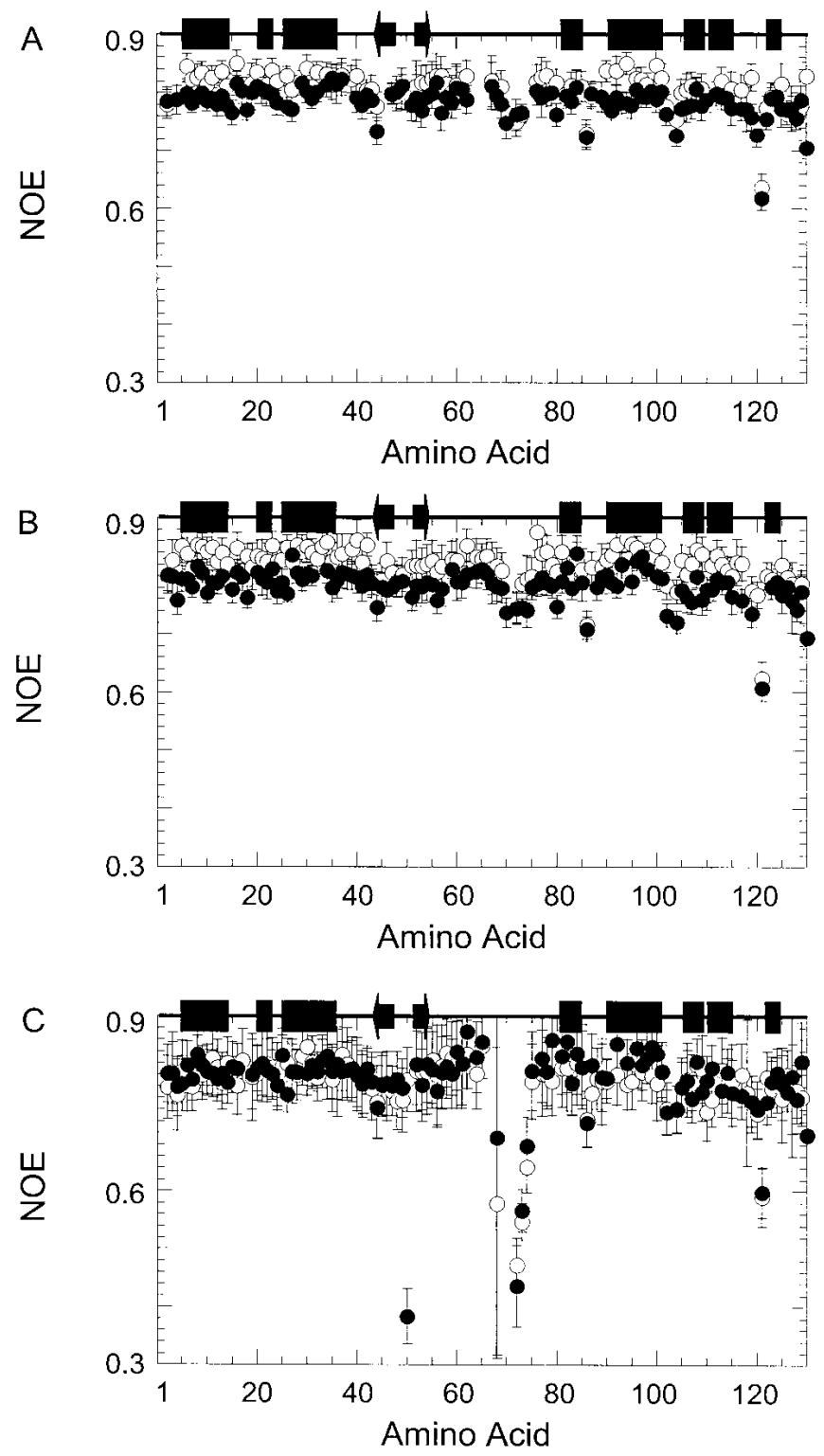

Fig. 2. ${ }^{1} \mathrm{H}-{ }^{15} \mathrm{~N}$ heteronuclear NOE values for $(A)$ wild-type, $(B)$ I56T, and (C) D67H human lysozymes. NOE values are shown at $20^{\circ} \mathrm{C}$ (open circles) and $37^{\circ} \mathrm{C}$ (closed circles). At the top of each figure the secondary structural elements are shown: helices (bars) and $\beta$-sheets (arrows). At $20^{\circ} \mathrm{C}$, the NOE value of R50 in D67H is 0.79 and is similar to its surrounding residues. At $37^{\circ} \mathrm{C}$, however, the measured value is only 0.38 . The overlap of resonances in the wild-type protein and I56T variant prevents the measurement of NOEs for this residue in these proteins. We cannot, therefore, account for this apparently low NOE value in $\mathrm{D} 67 \mathrm{H}$ at $37^{\circ} \mathrm{C}$.

The largest changes are in the long loop (residues 55-80) of the $\beta$-domain containing the mutation; in contrast, the dynamics of the residues in the $\alpha$-domain are closely similar to those of this domain in the wild-type protein. The NOE values of residues $\mathrm{G} 72, \mathrm{~A} 73$, and V74 decrease from $\sim 0.8$ to $0.44,0.56$, and 0.68 , respectively. The decreased NOE values indicate that the rapid (picosecond) backbone dynamics 
of these residues have increased considerably as a result of the change in amino acid at position 67 . These three residues are located immediately after the residue P71 that shows the largest change in $\mathrm{C}_{\alpha}$ position (9.9 $\AA$ ) between the wild-type and D67H crystal structures (Fig. 3). In addition, the low NMR peak intensity indicates that the line widths of residues close in sequence to $\mathrm{H67}$ (Y63, N66, H67, G68, K69, and T70) are substantially increased. These results indicate, therefore, that, in addition to the increases in the fast-time-scale dynamics of residues G72-V74, other residues close to the site of the mutation have developed complex dynamics that include much slower, millisecond to microsecond time-scale, motions that result in increased line widths.

\section{Conclusions}

Previous studies have shown that the two amyloidogenic variants of lysozyme investigated here have crystal structures similar to that of the wild-type protein and are enzymatically active (Booth et al. 1997). Both variants, however, have been found to have reduced stability relative to the wild-type protein as revealed by lower midpoints of thermal denaturation and by increased rates of hydrogen exchange (Booth et al. 1997; Canet et al. 1999). The lower stability of $\mathrm{D} 67 \mathrm{H}$ is almost entirely the result of a 160-fold increase in the unfolding rate compared to the wild-type protein, but I56T loses stability through a combined effect on both the unfolding and refolding rates. Although the origins of the reduced stabilities are therefore different, both variants have an increased equilibrium population of one or more species



Fig. 3. Ribbon diagram of the crystal structure of D67H lysozyme produced with Rasmol and the PDB file 1LYY (Booth et al. 1997). The $\alpha$ - and $\beta$-domains are in the lower right and upper left sides. The mutation sites, I56T and $\mathrm{D} 67 \mathrm{H}$, are shown in space-filling representations. The molecule is red in regions showing greatly altered chemical shifts (G48-D52 and T60-S82) and blue where reduced NOE values were measured (G72-V74). that are at least partially unfolded. These results have led to the hypothesis that the amyloidogenic nature of the variants arises from the increased population of such species that are likely to be prone to aggregation (Canet et al. 1999; Morozova-Roche et al. 2000). The present results support this hypothesis as they indicate that the native state dynamics of the protein do not correlate with the amyloidogenic behavior.

Despite the fact, therefore, that the native state of the D67H variant has a region close to the mutation site that has very high flexibility compared to the remainder of the protein, the absence of a region with similar flexibility in the I56T variant indicates that this is not by itself influential in stimulating the process of aggregation. Instead, this finding indicates that the conversion of soluble protein to amyloid fibrils requires a conformational transition in the protein that is significantly larger than the very rapid fluctuations that result in perturbations to NMR relaxation rates of the type observed for $\mathrm{D} 67 \mathrm{H}$. In the initial stages of aggregation, therefore, it is likely that a sufficiently large segment of the polypeptide chain must become exposed to the solvent in order to permit stable intermolecular interactions to develop. Important support for this conclusion has come very recently from the results of a mass spectrometric study of the hydrogen exchange properties of D67H lysozyme. This study reveals that highly cooperative fluctuations leading to the simultaneous exposure of a substantial region of the $\beta$-domain can, indeed, occur under conditions that favor the formation of amyloid structures (Canet et al., in prep.). Therefore, although mutations in the structure of lysozyme can result in changes both to the intrinsic dynamics of the native structure and to the ability to access substantially unfolded conformations, it is the latter that appears to be the origin of the amyloidogenic nature of the disease-related variants.

\section{Materials and methods}

Proteins labeled with ${ }^{15} \mathrm{~N}$ were expressed in Aspergillus niger and purified as described previously (Spencer et al. 1999). Protein samples were typically $1 \mathrm{mM}$, in $90 \% \mathrm{H}_{2} \mathrm{O} / 10 \% \mathrm{D}_{2} \mathrm{O}$ with the $\mathrm{pH}$ adjusted to 5.0 with $\mathrm{HCl}$ or $\mathrm{NaOH}$.

The wild-type and $\mathrm{D} 67 \mathrm{H}$ spectra were assigned by comparing HSQC spectra to previously published spectra of wild-type human lysozyme (Boyd et al. 1985; Redfield and Dobson 1990; Ohkubo et al. 1991) and confirmed with 2D COSY and 3D ${ }^{15} \mathrm{~N}-\mathrm{NOESY}-$ HSQC experiments. I56T spectra were assigned by comparing HSQC and COSY spectra to the wild-type spectra. Felix (Biosym Technologies) and Azara (Wayne Boucher, Cambridge) were used to process the spectra. Peak positions were determined from gradient-enhanced HSQC spectra (Kay et al. 1992) using an in-house algorithm. Tables with the ${ }^{1} \mathrm{HN}$ and ${ }^{15} \mathrm{~N}$ assignments are available as electronic supplementary material.

The ${ }^{1} \mathrm{H}-{ }^{15} \mathrm{~N}$ heteronuclear NOE values were calculated as the ratio of peak heights in spectra recorded with and without ${ }^{1} \mathrm{H}$ saturation (Kay et al. 1989). Presaturation was accomplished with $225^{\circ}{ }^{1} \mathrm{H}$ pulses applied with 5-msec intervals during the 5-sec 
recycle delay. Spectra were recorded on home-built $600-\mathrm{MHz}$ spectrometers belonging to the Oxford Centre for Molecular Sciences. The uncertainties in the NOE values were estimated by a comparison of duplicate experiments with the wild-type protein at $20^{\circ} \mathrm{C}$. The peak intensities and background noise were measured using the Felix macro scripts of M. Akke (Lund University, Sweden) and in-house programs, respectively. The chemical shift values of the backbone ${ }^{15} \mathrm{~N}$ and ${ }^{1} \mathrm{HN}$ resonances of the wild-type, I56T, and D67H lysozymes have been deposited in the BMRB with accession numbers 5123, 5124, and 5125 .

The chemical shift values of the backbone ${ }^{15} \mathrm{~N}$ and ${ }^{1} \mathrm{HN}$ resonances of the wild-type, I56T, and D67H lysozymes have been deposited in the BMRB with accession numbers 5123, 5124, and 5125 , respectively.

\section{Acknowledgments}

We are grateful to Jonathan Boyd, Jonathan Jones, and Denis Canet for assistance and valuable discussions. A.K.C. acknowledges receipt of a Helen Hay Whitney Foundation postdoctoral fellowship, V.R. an EMBO postdoctoral fellowship, and C.R. a BBSRC Advanced Research Fellowship. The research of C.M.D. is supported in part by a Programme Grant from the Wellcome Trust. This is a contribution from the Oxford Centre for Molecular Sciences, which is supported by the UK BBSRC, EPSRC, and MRC.

The publication costs of this article were defrayed in part by payment of page charges. This article must therefore be hereby marked "advertisement" in accordance with 18 USC section 1734 solely to indicate this fact.

\section{References}

Booth, D.R., Sunde, M., Bellotti, V., Robinson, C.V., Hutchinson, W.L., Fraser, P.E., Hawkins, P.N., Dobson, C.M., Radford, S.E., Blake, C.C.F., and Pepys, M.B. 1997. Instability, unfolding and aggregation of human lysozyme variants underlying amyloid fibrillogenesis. Nature 385: 787-793.

Boyd, J., Dobson, C.M., and Redfield, C. 1985. Assignment of resonances in the ${ }^{1} \mathrm{H}$ NMR spectrum of human lysozyme. Eur. J. Biochem. 153: 383-396.

Buck, M., Boyd, J., Redfield, C., MacKenzie, D.A., Jeenes, D.J., Archer, D.B., and Dobson, C.M. 1995. Structural determinants of protein dynamics: Analysis of ${ }^{15} \mathrm{~N}$ NMR relaxation measurements for main-chain and sidechain nuclei of hen egg white lysozyme. Biochemistry 34: 4041-4055.

Canet, D., Sunde, M., Last, A.M., Miranker, A., Spencer, A., Robinson, C.V., and Dobson, C.M. 1999. Mechanistic studies of the folding of human lysozyme and the origin of amyloidogenic behavior in its disease-related variants. Biochemistry 38: 6419-6427.
Chiti, F., Webster, P., Taddei, N., Cark, A., Stefani, M., Ramponi, G., and Dobson, C.M. 1999. Designing conditions for in vitro formation of amyloid protofilaments and fibrils. Proc. Natl. Acad. Sci. 96: 3590-3594.

Collaborative Computational Project 4, 1994. The CCP4 suite: Programs for protein crystallography. Acta Cryst. D50: 760-763.

Dobson, C.M. 2001. The structural basis of protein folding and its links with human disease. Phil. Trans. Roy. Soc. Lond. B Biol. Sci. 356: 133-145.

Eliezer, D., Yao, J., Dyson, H.J., and Wright, P.E. 1998. Structural and dynamic characterization of partially folded states of apomyoglobin and implications for protein folding. Nat. Struct. Biol. 5: 148-155.

Funahashi, J., Takano, K., Ogasahara, K., Yamagata, Y., and Yutani, Y. 1996. The structure, stability, and folding process of amyloidogenic mutant human lysozyme. J. Biochem. (Tokyo) 120: 1216-1223.

Kay, L.E., Torchia, D.A., and Bax, A. 1989. Backbone dynamics of proteins as studied by ${ }^{15} \mathrm{~N}$ inverse detected heteronuclear NMR spectroscopy: Application to staphylococcal nuclease. Biochemistry 28: 8972-8979.

Kay, L.E., Keifer, P., and Saarinen, T. 1992. Pure absorption gradient enhanced heteronuclear single quantum correlation spectroscopy with improved sensitivity. J. Amer. Chem. Soc. 114: 10663-10665.

Koo, E.H., Lansbury, P.T., Jr., and Kelly, J.W. 1999. Amyloid diseases: Abnormal protein aggregation in neurodegeneration. Proc. Natl. Acad. Sci. 96: 9989-9990.

Lansbury, P.T., Jr. 1999. Evolution of amyloid: What normal protein folding may tell us about fibrillogenesis and disease. Proc. Natl. Acad. Sci. 96: 3342-3344.

Morozova-Roche, L.A., Zurdo, J., Spencer, A., Noppe, W., Receveur, V., Archer, D.B., Joniau, M., and Dobson, C.M. 2000. Amyloid fibril formation and seeding by wild-type human lysozyme and its disease-related mutational variants. J. Struct. Biol. 130: 339-351.

Ohkubo, T., Taniyama, Y., and Kikuchi, M. 1991. ${ }^{1} \mathrm{H}$ and ${ }^{15} \mathrm{~N}$ NMR study of human lysozyme. J. Biochem. 110: 1022-1029.

Pepys, M.B. 1994. Amyloidosis. In Samter's Immunological Diseases (eds. M.M. Frank et al.), pp. 631-674. Little, Brown and Company, Boston.

Pepys, M.B., Hawkins, P.N., Booth, D.R., Vigushin, D.M., Tennent, G.A., Soutar, A.K., Totty, N., Nguyen, O., Blake, C.C., Terry, C.J., et al. 1993. Human lysozyme gene mutations cause hereditary systemic amyloidosis. Nature 362: 553-557.

Prusiner, S.B. 1997. Prion diseases and the BSE crisis. Science 278: 245-251.

Redfield, C. and Dobson, C.M. 1990. ${ }^{1} \mathrm{H}$ NMR studies of human lysozyme: Spectral assignment and comparison with hen lysozyme. Biochemistry 29: 7201-7214.

Serpell, L.C., Sunde, M., and Blake, C.C. 1997. The molecular basis of amyloidosis. Cell. Mol. Life Sci. 53: 871-887.

Spencer, A., Morozov-Roche, L.A., Noppe, W., MacKenzie, D.A., Jeenes, D.J., Joniau, M., Dobson, C.M., and Archer, D.B. 1999. Expression, purification, and characterization of the recombinant calcium-binding equine lysozyme secreted by the filamentous fungus Aspergillus niger: Comparisons with the production of hen and human lysozymes. Prot. Exp. Purif. 16: 171-180.

Sunde, M. and Blake, C.C.F. 1998. From the globular to the fibrous state: Protein structure and structural conversion in amyloid formation. Quar. Rev. Biophys. 31: 1-39.

Wüthrich, K. 1986. NMR of proteins and nucleic acids. John Wiley, New York. 